\title{
Regularity of minimizers of quasi perimeters with a volume constraint
}

\author{
QINGLAN XIA ${ }^{\dagger}$ \\ University of Texas at Austin, Mathematics, Austin, TX 78741, USA
}

[Received 16 September 2004 and in revised form 1 March 2005]

\begin{abstract}
We study the regularity of the boundary of sets minimizing a quasi perimeter $\mathbf{T}(E)=P(E, \Omega)+$ $\mathbf{G}(E)$ with a volume constraint. Here $\Omega$ is any open subset of $\mathbb{R}^{n}$ with $n \geqslant 2$, and $\mathbf{G}$ is a lower semicontinuous function on sets of finite perimeter satisfying $\mathbf{G}(E) \leqslant \mathbf{G}(F)+C|E \triangle F|^{\beta}$ for all sets $E, F$ of finite perimeter with equal volume. We show that under the condition $\beta>1-1 / n$, any volume constrained minimizer $E$ of the quasi perimeter $\mathbf{T}$ has both interior points and exterior points, and $E$ is indeed a quasi minimizer of perimeter without the volume constraint. Using a well known regularity result for quasi minimizers of perimeter, we get the classical $C^{1, \alpha}$ regularity for the reduced boundary of $E$.
\end{abstract}

\section{Introduction}

Let $\Omega \subset \mathbb{R}^{n}$ be any open subset with $n \geqslant 2$. We consider the following minimization problem: Minimize

$$
\mathbf{T}(E)=P(E, \Omega)+\mathbf{G}(E)
$$

among all sets $E \subset \Omega$ of finite perimeter with a fixed volume.

Here $P(E, \Omega)$ denotes the perimeter of $E$, and $\mathbf{G}$ is a lower semicontinuous functional on the sets of finite perimeter in $\Omega$ with the property that

$$
\mathbf{G}(E) \leqslant \mathbf{G}(F)+C|E \triangle F|^{\beta}
$$

for any sets $E, F$ in $\Omega$ of finite perimeter with $|E|=|F|$, for some constant $C>0$ and a number $\beta>1-1 / n$. Here $E \triangle F$ is the symmetric difference $(E \backslash F) \cup(F \backslash E)$.

The special case that $\mathbf{G}(E) \equiv 0$ corresponds to a well known problem which concerns sets minimizing perimeter with a volume constraint. This problem is often encountered in capillarity theory. Liquid drops, resting on or hanging from a given surface, are typical examples. The regularity of the corresponding minimizers has been studied extensively in [2].

Another example is given by

$$
\mathbf{G}(E)=\int_{E} H(x) \mathrm{d} x
$$

where $H \in L^{p}(\Omega)$, for some $p>n$, is a given function. Without a volume constraint, this is the problem of finding sets with prescribed mean curvature $H$, and has been studied for instance in [3] by Massari. In our case, we impose an additional volume constraint on it. From the Hölder inequality, we see that $\beta=1-1 / p>1-1 / n$ here.

$\dagger$ This work was supported by an NSF grant DMS-0306686. The author wants to express his thanks to Professor Luis Caffarelli and Robert Hardt for helpful discussions. E-mail: qlxia@math.utexas.edu 
Our main motivation for considering this problem comes from the study of mud cracking and related problems. Mud cracking represents a very typical physical phenomenon. After losing a certain amount of moisture, a material such as a piece of mud will begin to crack. People are interested in why, how and where the material cracks. To understand these problems, we propose the following variational model. Let $\Omega$ represent a piece of mud. After losing a certain amount of moisture, say $\sigma|\Omega|$ for some $\sigma \in(0,1)$, the volume of the mud decreases, and thus a crack $E$ of volume $\sigma|\Omega|$ must come out to replace the losing volume. The selection of cracking is not totally random, but the actual physics of it might be too complicated to handle. Instead, we may assume that it minimizes the total work of transporting the old mud $\Omega$ to the new mud $\Omega \backslash E$, with multiplicity $1 /(1-\sigma)$, under a volume preserving map. To justify this idea, let us think about a mud in the shape of a disk. To replace the volume of losing moisture, it can either shrink evenly to a smaller disk or dig some space out by cracking inside it. Which way is better? As we know, the mud will possibly choose the latter way. This is because the corresponding transport costs of two ways are different. The mud just chooses a cheaper way to reduce the total work. A reasonable way to represent the total work is given by the Wasserstein distances $W_{p}$ between Radon measures of equal total mass for some $p>0$. We refer to [5, Chapter 7] for the concepts of Wasserstein distances and related topics. As a result, one would like to minimize

$$
W_{p}^{q}\left(\mathcal { L } ^ { n } \left\lfloor\Omega, \frac{1}{1-\sigma} \mathcal{L}^{n}\lfloor(\Omega \backslash E))+P(E, \Omega)\right.\right.
$$

among all sets $E$ of finite perimeter in $\Omega$ with volume $|E|=\sigma|\Omega|$ for some $\sigma \in(0,1)$. Here, $q=\max (1, p), \mathcal{L}^{n}\left\lfloor K\right.$ denotes the Lebesgue measure $\mathcal{L}^{n}$ restricted to any measurable set $K$, and the perimeter $P(E, \Omega)$ of $E$ is used to represent the cracking energy for breaking the mud. Using the properties of Wasserstein distances, it is easy to see that

$$
W_{p}\left(\mathcal { L } ^ { n } \left\lfloor\Omega, \frac{1}{1-\sigma} \mathcal{L}^{n}\lfloor(\Omega \backslash E))=W_{p}\left(\frac { 1 } { 1 - \sigma } \mathcal { L } ^ { n } \left\lfloorE, \frac{\sigma}{1-\sigma} \mathcal{L}^{n}\lfloor\Omega)=\lambda W_{p}\left(\mathcal { L } ^ { n } \left\lfloorE, \sigma \mathcal{L}^{n}\lfloor\Omega)\right.\right.\right.\right.\right.\right.
$$

for some constant $\lambda>0$. Thus, the problem amounts to minimizing

$$
P(E, \Omega)+\lambda W_{p}^{q}\left(\mathcal { L } ^ { n } \left\lfloorE, \sigma \mathcal{L}^{n}\lfloor\Omega)\right.\right.
$$

among all sets $E$ in $\Omega$ of finite perimeter and with a volume constraint $|E|=\sigma|\Omega|$. In this case,

$$
\mathbf{G}(E)=\lambda W_{p}^{q}\left(\mathcal { L } ^ { n } \left\lfloorE, \sigma \mathcal{L}^{n}\lfloor\Omega) .\right.\right.
$$

It is easy to see that $\beta=1$ here.

Keeping all these examples in mind, we would like to study the minimizers for more general $\mathbf{G}$. Note that the existence of minimizers for the quasi perimeter $\mathbf{T}$ follows immediately from the compactness of sets of finite perimeter. Therefore, this article is mainly focused on the regularity of these minimizers. Further properties as well as numerical simulation will be considered elsewhere. The special case $\mathbf{G}(E) \equiv 0$ was studied in [2]. The approach there was to show that volume constrained minimizers are quasi perimeter minimizing in small balls without the volume constraint. This yields regularity results analogous to those for the unconstrained problem. A key step there is showing the existence of interior and exterior points of the minimizer. Here we adopt the same approach as in [2]. That is, we show that these $\mathbf{T}$ minimizers indeed have both interior points and exterior points (see Theorem 4.3), and in fact they are quasi minimizers of perimeter without the 
volume constraint (see Theorem 4.4). Then we use the known results of [4, Theorem 1] about quasi minimizers of perimeter to get the desired regularity of these $\mathbf{T}$ minimizers.

The paper is organized as follows. After providing some basic notations about perimeters, we provide an estimate on how fast the infimum of the metric density is approaching 0 for any set of finite perimeter. Using this estimate and some technical lemmas, we classify the sets of finite perimeter into two classes (see Corollary 3.4 for details). Using this classification and also properties of $\mathbf{T}$ minimizers, we show that any $\mathbf{T}$ minimizer will have both interior points and exterior points in $\Omega$. By this result, we get rid of the volume constraint and prove our main theorems in Section 4 .

\section{Preliminaries}

Here we only mention the basic notations and definitions about perimeters.

We assume that $\Omega$ is an open (bounded) subset of $\mathbb{R}^{n}$ with $n \geqslant 2$. If $E \subseteq \Omega,|E|$ is the Lebesgue measure of $E$, and $\chi_{E}(x)$ is the characteristic function of $E ; \mathcal{H}^{s}(\cdot)$ denotes the $s$-dimensional Hausdorff measure. Finally, $E^{c}$ is the complement of $E$ in $\Omega$.

Recall that a function $f \in L^{1}(\Omega)$ is of bounded variation in $\Omega$ if

$$
\|D f\|(\Omega)=\sup \left\{\int_{\Omega} f \operatorname{div} \phi \mathrm{d} x: \phi \in C_{0}^{1}\left(\Omega, \mathbb{R}^{n}\right),|\phi|(x) \leqslant 1\right\}<\infty .
$$

A set $E \subset \Omega$ is said to be of finite perimeter in $\Omega$ if its characteristic function $\chi_{E}$ is of bounded variation in $\Omega$. We will use the notation $P(E, \Omega)$ for the perimeter so that

$$
P(E, \Omega)=\left\|D \chi_{E}\right\|(\Omega) .
$$

For $\partial E \cap \Omega$ sufficiently smooth, $P(E, \Omega)=\mathcal{H}^{n-1}(\partial E \cap \Omega)$.

Let $\alpha=1-1 / n$. For any set $E$ of finite perimeter in $\Omega$, the isoperimetric inequality says that

$$
P(E, \Omega) \geqslant C_{n}|E|^{\alpha},
$$

where $C_{n}=n(\alpha(n))^{1 / n}$ with $\alpha(n)$ being the Lebesgue measure of the unit ball in $\mathbb{R}^{n}$. Moreover, for each $n$-dimensional open cube $Q \subset \Omega$, the relative isoperimetric inequality says that

$$
\gamma(n) P(E, Q) \geqslant \min \left\{|E \cap Q|^{\alpha},\left|E^{c} \cap Q\right|^{\alpha}\right\}
$$

for some constant $\gamma(n)>0$.

In the following, we shall frequently use some simple properties of sets of finite perimeter, which can be found for instance in [1]. Here, we also mention a property that we will use later. Let $B$ be any open ball of radius $r$. For every set $L$ of finite perimeter in $B$, we have (see [2, (8)])

$$
\mathcal{H}^{n-1}(L \cap \partial B) \leqslant P(L, B)+\frac{n}{r}|L \cap B|
$$

in the sense of traces.

\section{Exterior points of sets of finite perimeter}

Let $E$ be a set of finite perimeter in $\Omega$. In this article, a point $p \in \Omega$ is said to be an exterior (or interior) point of $E$ if there exists an open ball neighborhood $B(p, r)$ of $p$ for some $r>0$ such that

$$
|E \cap B(p, r)|=0 \quad(\text { or }|E \cap B(p, r)|=|B(p, r)|, \text { respectively }) .
$$


If $|E|=0$, then every point is an exterior point of $E$, while if $|E|=|\Omega|$, then every point is an interior point of $E$. In general, if $0<|E|<|\Omega|, E$ may not have exterior or interior points in $\Omega$. Their existence becomes an interesting problem to study.

To study the existence of exterior points, we consider the following function. For any $r \geqslant 0$, let

$$
f(r)=\inf _{x \in \Omega}|E \cap Q(x, r)|,
$$

where $Q(x, r)$ denotes the $n$-dimensional open cube in $\mathbb{R}^{n}$ centered at $x$ and with edge length $r$. Note that $f(0)=0$ and $f(r)$ is an increasing function of $r$. Also, $E$ has exterior points in $\Omega$ if and only if $f(r) \equiv 0$ in a small neighborhood of 0 .

For any point $p$ in $\Omega$ with metric density 0 , one may see directly that

$$
|E \cap B(p, r)|=o\left(r^{n}\right),
$$

and thus $f(r)=o\left(r^{n}\right)$ as $r$ approaches 0 . This is true even if $E$ is not of finite perimeter. When $E$ is indeed a set of finite perimeter, we can get a better result $f(r)=o\left(r^{n+1}\right)$, which is demonstrated by the following theorem.

THEOREM 3.1 Suppose $E$ is a set of finite perimeter in $\Omega$ with $|E|<|\Omega|$. Then there exists an $\eta>0$ such that for any $r \in[0, \eta)$,

$$
0 \leqslant f(r) \leqslant C_{1} r^{n^{2} /(n-1)}
$$

for some constant $C_{1} \geqslant 0$, depending on $E$.

Proof. Let $p \in \Omega \backslash E$ be any point with metric density 0 , that is,

$$
\lim _{r \rightarrow 0} \frac{|E \cap B(p, r)|}{\alpha_{n} r^{n}}=0 .
$$

Thus, there exists an $\eta_{1}>0$ such that

$$
\left|Q\left(p, \eta_{1}\right) \cap E\right| \leqslant \eta_{1}^{n} / 4 .
$$

Now, for any $r \leqslant \eta_{1}$, one can subdivide $Q=Q\left(p, \eta_{1}\right)$ into $\left[\eta_{1} / r\right]^{n}$ disjoint smaller cubes $\left\{Q_{j}\right\}$ with edge length $r$, where $[x]$ denotes the integer part of $x$. Let

$$
\mathbf{A}=\left\{Q_{j}:\left|E \cap Q_{j}\right|>\frac{1}{2} r^{n}\right\}, \quad \mathbf{B}=\left\{Q_{j}:\left|E \cap Q_{j}\right| \leqslant \frac{1}{2} r^{n}\right\} .
$$

Then $|\mathbf{A}|+|\mathbf{B}|=\left[\eta_{1} / r\right]^{n}$ and

$$
\frac{1}{2}|\mathbf{A}| r^{n} \leqslant \sum_{Q_{j} \in \mathbf{A}}\left|E \cap Q_{j}\right| \leqslant|E \cap Q| \leqslant \frac{1}{4} \eta_{1}^{n},
$$

where $|\mathbf{A}|$ denotes the number of elements in the set $\mathbf{A}$. Thus, $|\mathbf{A}| \leqslant \frac{1}{2}\left(\eta_{1} / r\right)^{n}$ and

$$
|\mathbf{B}|=\left[\frac{\eta_{1}}{r}\right]^{n}-|\mathbf{A}| \geqslant\left[\frac{\eta_{1}}{r}\right]^{n}-\frac{1}{2}\left(\frac{\eta_{1}}{r}\right)^{n} \geqslant \frac{1}{4}\left(\frac{\eta_{1}}{r}\right)^{n}
$$

if $r<(1-\sqrt[n]{0.75}) \eta_{1}$ 
By the relative isoperimetric inequality, for any $Q_{i} \in \mathbf{B}$,

$$
\left|E \cap Q_{i}\right|^{1-1 / n} \leqslant \gamma(n) P\left(E, Q_{i}\right)
$$

for some constant $\gamma(n)$. Therefore,

$$
\begin{aligned}
|\mathbf{B}| f(r)^{1-1 / n} & =\sum_{Q_{i} \in \mathbf{B}} f(r)^{1-1 / n} \leqslant \sum_{Q_{i} \in \mathbf{B}}\left|E \cap Q_{i}\right|^{1-1 / n} \\
& \leqslant \sum_{Q_{i} \in \mathbf{B}} \gamma(n) P\left(E, Q_{i}\right) \leqslant \gamma(n) P(E, Q)<\infty
\end{aligned}
$$

because $E$ has finite perimeter in $Q$. Thus,

$$
f(r) \leqslant\left(\frac{\gamma(n) P(E, Q)}{|\mathbf{B}|}\right)^{n /(n-1)} \leqslant\left(\frac{4 \gamma(n) P(E, Q)}{\left(\eta_{1}\right)^{n}}\right)^{n /(n-1)} r^{n^{2} /(n-1)}
$$

whenever $r \leqslant \eta=(1-\sqrt[n]{0.75}) \eta_{1}$.

Proposition 3.2 Suppose $E$ is a set of finite perimeter in $\Omega$ with $|E|<|\Omega|$, and $\tau$ is any positive real number. Let $Q$ be any $n$-dimensional open cube in $\Omega$ with edge length $r$ satisfying

$$
r<\left(\frac{\tau}{2^{n+2}}\right)^{n-1} \frac{1}{\left(2 C_{1}\right)^{\alpha}}
$$

and

$$
|E \cap Q| \leqslant 2 C_{1} r^{n^{2} /(n-1)} .
$$

Then there exists an $s \in[r / 2, r]$ such that

$$
|E \cap Q(p, s)| \leqslant 2 C_{1} s^{n^{2} /(n-1)}
$$

and

$$
\mathcal{H}^{n-1}(E \cap \partial Q(p, s)) \leqslant \tau|E \cap Q(p, s)|^{\alpha},
$$

where $Q(p, s)$ is the cube having the same center $p$ of $Q$ and with edge length $s$.

Proof. We consider the function $g:[0, r] \rightarrow[0, \infty)$ defined by

$$
g(s)=|E \cap Q(p, s)| \quad \text { for } s \in[0, r] .
$$

Then $g(0)=0$ and

$$
g^{\prime}(s)=\mathcal{H}^{n-1}(E \cap \partial Q(p, s)) \quad \text { for almost all } s .
$$

From (3.1) and (3.2), we have

$$
\int_{0}^{r}\left(g(s)^{1 / n}\right)^{\prime} \mathrm{d} s=g(r)^{1 / n}-g(0)^{1 / n} \leqslant\left(2 C_{1}\right)^{1 / n} r^{n /(n-1)} \leqslant \frac{\tau}{n} r .
$$

Therefore, there exists an $s \in[0, r]$ such that $\left(g(s)^{1 / n}\right)^{\prime} \leqslant \tau / n$. That is,

$$
g^{\prime}(s) \leqslant \tau g(s)^{\alpha} .
$$


Let

We claim that

$$
s_{0}=\max \left\{s \in[0, r]: g^{\prime}(s) \leqslant \tau g(s)^{\alpha}\right\} \leqslant r .
$$

$$
g\left(s_{0}\right) \leqslant 2 C_{1} s_{0}^{n^{2} /(n-1)} .
$$

In fact, if $s_{0}=r$, then this follows from our assumption 3.2). If $s_{0}<r$, then for any $s \in\left(s_{0}, r\right)$, we have $g^{\prime}(s)>\tau g(s)^{\alpha}$, which yields $\left(g(s)^{1 / n}\right)^{\prime}>\tau / n$. Integrating it from $s_{0}$ to $r$ yields

$$
g(r)^{1 / n}-g\left(s_{0}\right)^{1 / n} \geqslant \frac{\tau}{n}\left(r-s_{0}\right) .
$$

Therefore,

$$
\begin{aligned}
g\left(s_{0}\right)^{1 / n} & \leqslant g(r)^{1 / n}-\frac{\tau}{n}\left(r-s_{0}\right) \leqslant\left(2 C_{1}\right)^{1 / n} r^{n /(n-1)}-\frac{\tau}{n}\left(r-s_{0}\right) \\
& \leqslant\left(2 C_{1}\right)^{1 / n} s_{0}^{n /(n-1)} .
\end{aligned}
$$

The last inequality follows from the fact that the function

$$
h(x)=\left(2 C_{1}\right)^{1 / n} x^{n /(n-1)}-\frac{\tau}{n} x
$$

is decreasing on $[0, r]$ because $h^{\prime}(x)=\left(2 C_{1}\right)^{1 / n} \frac{n}{n-1} x^{1 /(n-1)}-\tau / n \leqslant 0$, by 3.1 .

Moreover, since $g\left(s_{0}\right) \geqslant 0$, by (3.3) and 3.1), we have

$$
r-s_{0} \leqslant \frac{n}{\tau} g(r)^{1 / n} \leqslant \frac{n}{\tau}\left(2 C_{1}\right)^{1 / n} r^{n /(n-1)} \leqslant \frac{r}{2} .
$$

Therefore, we have $s_{0} \geqslant r / 2$. This $s_{0}$ is the desired $s$.

From now on, we let $\delta$ be a number such that

$$
0<\delta \leqslant 4^{-n^{2} /(n-1)} .
$$

Let

$$
0<\tau \leqslant \frac{1}{4}\left(2-2^{\alpha}\right) C_{n} \delta^{\alpha}
$$

it is easy to check that

$$
\tau \leqslant \frac{1}{4} C_{n}
$$

Let $A_{\tau}(E)$ be the family of all $n$-dimensional open cubes in $\Omega$ satisfying

$$
|E \cap Q| \leqslant 2 C_{1} r^{n^{2} /(n-1)}, \quad \mathcal{H}^{n-1}(E \cap \partial Q) \leqslant \tau|E \cap Q|^{\alpha},
$$

where $r$ is the edge length of $Q$ satisfying 3.1 .

Proposition 3.3 Let $E$ be a set of finite perimeter with $|E|<|\Omega|$, and $Q$ be any cube in $A_{\tau}(E)$. Then either

$$
P(E, Q) \geqslant\left(C_{n}+2 \tau\right)|E \cap Q|^{\alpha}
$$

or there exists a smaller cube $\tilde{Q} \subset Q$ such that $\tilde{Q} \in A_{\tau}(E)$ and the edge length $\tilde{r}$ of $\tilde{Q}$ satisfies

$$
\tilde{r} \in(r / 8,3 r / 4) .
$$


Proof. Without losing generality, we may assume that $Q$ is centered at the origin $O$. Let

$$
Z=\left\{s \in\left(-\frac{r}{2}, \frac{r}{2}\right): \sum_{i=1}^{n} \mathcal{H}^{n-1}\left(E \cap\left\{x=\left(x_{1}, \ldots, x_{n}\right) \in Q: x_{i}=s\right\}\right) \geqslant \frac{\tau}{2}|E \cap Q|^{\alpha}\right\} .
$$

Then, since

$$
\begin{aligned}
n|E \cap Q| & \geqslant \sum_{i=1}^{n} \int_{-r / 2}^{r / 2} \mathcal{H}^{n-1}\left(E \cap\left\{x \in Q: x_{i}=s\right\}\right) \mathrm{d} s \\
& \geqslant \int_{Z} \sum_{i=1}^{n} \mathcal{H}^{n-1}\left(E \cap\left\{x \in Q: x_{i}=s\right\}\right) \mathrm{d} s \geqslant \frac{\tau}{2}|E \cap Q|^{\alpha} \mathcal{H}^{1}(Z),
\end{aligned}
$$

by applying 3.1) and 3.2), we have

$$
\mathcal{H}^{1}(Z) \leqslant \frac{2 n}{\tau}|E \cap Q|^{1 / n}<\frac{2 n}{\tau}\left(2 C_{1}\right)^{1 / n} r^{n /(n-1)}<\frac{r}{2} .
$$

Therefore, there exists $s_{0} \in(-r / 4, r / 4)$ such that

$$
\sum_{i=1}^{n} \mathcal{H}^{n-1}\left(E \cap\left\{x=\left(x_{1}, \ldots, x_{n}\right) \in Q: x_{i}=s_{0}\right\}\right)<\frac{\tau}{2}|E \cap Q|^{\alpha} .
$$

Using the hyperplanes $\left\{x: x_{i}=s_{0}\right\}$, we decompose $Q$ into the union of $2^{n}$ smaller $n$-dimensional rectangles $\left\{Q_{1}, Q_{2}, \ldots, Q_{2^{n}}\right\}$. Each $Q_{i}$ is located in one corner of $Q$, and two of these rectangles are in fact $n$-dimensional cubes with edge lengths $r / 2 \pm s_{0}$. Since $s_{0} \in(-r / 4, r / 4)$, we have

$$
r / 4<r / 2 \pm s_{0}<3 r / 4 .
$$

Now, if

$$
\max _{i}\left|E \cap Q_{i}\right| \leqslant(1-\delta)|E \cap Q|,
$$

then we can rearrange $\left\{Q_{i}\right\}$ into two groups

$$
V_{1}=\bigcup\left\{\bar{Q}_{i_{1}}, \bar{Q}_{i_{2}}, \ldots, \bar{Q}_{i_{k}}\right\} \quad \text { and } \quad V_{2}=Q \backslash V_{1}
$$

such that

$$
\delta|E \cap Q| \leqslant\left|E \cap V_{i}\right| \leqslant(1-\delta)|E \cap Q|
$$

for each $i=1,2$. A well known inequality says

$$
\left(2-2^{\alpha}\right)(\min (a, b))^{\alpha} \leqslant a^{\alpha}+b^{\alpha}-(a+b)^{\alpha} \quad \text { for any } a, b \geqslant 0 .
$$

Therefore,

$$
\begin{aligned}
\left|E \cap V_{1}\right|^{\alpha}+\left|E \cap V_{2}\right|^{\alpha}-|E \cap Q|^{\alpha} & \geqslant\left(2-2^{\alpha}\right) \min \left\{\left|E \cap V_{1}\right|^{\alpha},\left|E \cap V_{2}\right|^{\alpha}\right\} \\
& \geqslant\left(2-2^{\alpha}\right) \delta^{\alpha}|E \cap Q|^{\alpha} \geqslant \frac{4 \tau}{C_{n}}|E \cap Q|^{\alpha} .
\end{aligned}
$$


Hence, by the isoperimetric inequality, 3.7, and 3.6,

$$
\begin{aligned}
P(E, Q) & \geqslant P\left(E, V_{1}\right)+P\left(E, V_{2}\right) \\
& \geqslant C_{n}\left|E \cap V_{1}\right|^{\alpha}+C_{n}\left|E \cap V_{2}\right|^{\alpha}-\mathcal{H}^{n-1}\left(E \cap \partial V_{1}\right)-\mathcal{H}^{n-1}\left(E \cap \partial V_{2}\right) \\
& \geqslant C_{n}\left|E \cap V_{1}\right|^{\alpha}+C_{n}\left|E \cap V_{2}\right|^{\alpha}-2 \frac{\tau}{2}|E \cap Q|^{\alpha}-\mathcal{H}^{n-1}(E \cap \partial Q) \\
& \geqslant C_{n}|E \cap Q|^{\alpha}+4 \tau|E \cap Q|^{\alpha}-\tau|E \cap Q|^{\alpha}-\tau|E \cap Q|^{\alpha} \\
& =\left(C_{n}+2 \tau\right)|E \cap Q|^{\alpha} .
\end{aligned}
$$

This gives the first case. If

$$
\max _{i}\left|E \cap Q_{i}\right|>(1-\delta)|E \cap Q|,
$$

then at least one of $Q_{i}$ 's, say $Q_{1}$, satisfies

$$
\left|E \cap Q_{1}\right|<\delta|E \cap Q| \leqslant \delta\left(2 C_{1}\right) r^{n^{2} /(n-1)} \leqslant \delta\left(2 C_{1}\right)\left(4 r_{1}\right)^{n^{2} /(n-1)} \leqslant 2 C_{1} r_{1}^{n^{2} /(n-1)}
$$

where $r_{1}=r / 2 \pm s_{0}$ is the edge length of $Q_{1}$. By Proposition 3.2, there exists a smaller cube $\tilde{Q} \subset Q_{1}$ such that $\tilde{Q} \in A_{\tau}(E)$ with edge length $\tilde{r} \in\left(r_{1} / 2, r_{1}\right) \subset(r / 8,3 r / 4)$, due to 3.8 . This completes the second part.

COROLlaRY 3.4 Let $E \subset \Omega$ be a set of finite perimeter with $|E|<|\Omega|$. Then one of the following two cases must be true:

(1) either for any $\lambda>0$, there exists a cube $Q \in A_{\tau}(E)$ with edge length $r<\lambda$ and

$$
P(E, Q) \geqslant\left(C_{n}+2 \tau\right)|E \cap Q|^{\alpha} ;
$$

(2) or there exists a sequence $\left\{Q_{i}\right\}$ of cubes in $A_{\tau}(E)$ such that $Q_{i+1} \subset Q_{i}$ and their edge lengths satisfy

$$
\frac{1}{8} r_{i} \leqslant r_{i+1} \leqslant \frac{3}{4} r_{i} \quad \text { for each } i
$$

Proof. Follows from Proposition 3.3 .

REMARK 3.5 In the second case of Corollary 3.4 we may define a family of open cubes as follows. Let $Q_{0}$ be any given cube in $\Omega$. By picking the first cube $Q_{1}$ inside $Q_{0}$, we get a sequence $\left\{Q_{i}\right\}_{i=1}^{\infty}$ of cubes as in the second case of Corollary 3.4. and all these smaller cubes are contained in $Q_{0}$. By rescaling and translation, each cube $Q_{i}$ is the image of $[-1,1]^{n}$ under some affine map $f_{i}$ for each $i=0,1, \ldots$ Using these affine maps, we define a continuous map $F:[-1,1]^{n} \times(0, \infty) \rightarrow \mathbb{R}^{n}$ by setting

$$
F(x, s)= \begin{cases}\frac{s}{r_{1}}\left(f_{0}(x)-f_{0}(0)\right)+f_{0}(0) & \text { if } s>r_{0}, \\ \frac{1}{r_{i}-r_{i+1}}\left(\left(s-r_{i+1}\right) f_{i}(x)+\left(r_{i}-s\right) f_{i+1}(x)\right) & \text { if } s \in\left[r_{i+1}, r_{i}\right],\end{cases}
$$

for some $i$. Note that, for each $s>0$, the image $F_{s}=F\left(Q_{0}, s\right)$ is also a cube with edge length $s$. Also, $F_{r_{i}}=Q_{i}$ for each $i=0,1,2, \ldots$, and $F_{s} \subseteq F_{t}$ whenever $s \leqslant t$. Moreover, if $s \in\left[r_{i+1}, r_{i}\right]$ for some $i=1,2, \ldots$, we have

$$
Q_{i+1} \subseteq F_{s} \subseteq Q_{i} \subset Q_{0}
$$


and

$$
\left|E \cap F_{s}\right| \leqslant\left|E \cap Q_{i}\right| \leqslant 2 C_{1} r_{i}^{n^{2} /(n-1)} \leqslant 2 C_{1}(8 s)^{n^{2} /(n-1)} .
$$

Therefore, $\left|E \cap F_{S}\right|$ is continuous in $s$ and

$$
\left|E \cap F_{S}\right| \leqslant C_{E} s^{n^{2} /(n-1)}
$$

for any $s \in\left(0, r_{1}\right)$, where $C_{E}=2 C_{1} 8^{n^{2} /(n-1)}$. Similarly, for any open ball $B$ in $\Omega$, we may pick the first cube $Q_{1}$ inside $B$, and then construct a family $\left\{F_{s}\right\}$ of cubes as above. If we set $K_{s}$ to be the largest open ball of diameter $s$ inscribed in the cube $F_{s}$, then we get a family $\left\{K_{s}\right\}$ of open balls such that $\left|K_{s} \cap E\right|$ is also continuous in $s$ with

$$
\left|E \cap K_{s}\right| \leqslant C_{E} s^{n^{2} /(n-1)} .
$$

\section{Minimizers of quasi perimeters}

Let $\Omega$ be any bounded open subset of $\mathbb{R}^{n}$ with $n \geqslant 2$. For any $\sigma \in(0,1)$, let

$$
\mathcal{F}_{\sigma}=\{E \subset \Omega: P(E, \Omega)<\infty,|E|=\sigma|\Omega|\} .
$$

For any $E \in \mathcal{F}_{\sigma}$, a quasi perimeter of $E$ is of the form

$$
\mathbf{T}(E)=P(E, \Omega)+\mathbf{G}(E),
$$

where $\mathbf{G}$ is a lower semicontinuous functional on $\mathcal{F}_{\sigma}$ with the property that

$$
\mathbf{G}(A) \leqslant \mathbf{G}(B)+C|A \triangle B|^{\beta}
$$

for any $A, B \in \mathcal{F}_{\sigma}$, for some constant $C>0$ and a number $\beta>1-1 / n$. Some examples of $\mathbf{G}$ may be found in the introduction.

\subsection{Existence of interior and exterior points}

Note that for any $\mathbf{G}$, by the compactness of sets of finite perimeter, the quasi perimeter $\mathbf{T}$ automatically has a minimizer. The following lemma says that for a $\mathbf{T}$ minimizer $E$, only the second case of Corollary 3.4 can happen.

Lemma 4.1 Let $E$ be any $\mathbf{T}$ minimizer in $\mathcal{F}_{\sigma}$. Then there exists a sequence $\left\{Q_{i}\right\}$ of cubes in $A_{\tau}(E)$ such that $Q_{i+1} \subset Q_{i}$ and their edge lengths satisfy

$$
\frac{1}{8} r_{i} \leqslant r_{i+1} \leqslant \frac{3}{4} r_{i} \quad \text { for each } i .
$$

Proof. This is trivial if $E$ has exterior points. Therefore, we may assume that $E$ has no exterior points in $\Omega$. Under this assumption, we will prove the result by showing that the first case in Corollary 3.4 will not happen.

Assume that there exists a cube $Q$ in $A_{\tau}(E)$ such that

$$
P(E, Q) \geqslant\left(C_{n}+2 \tau\right)|E \cap Q|^{\alpha}
$$


and its edge length $r$ satisfies

$$
r<\lambda=\left(\frac{\tau}{2^{\beta} C\left(2 C_{1}\right)^{\beta-\alpha}}\right)^{\frac{n-1}{n^{2}(\beta-\alpha)}} .
$$

Then we consider another set

$$
\tilde{E}=(E \backslash Q) \cup B,
$$

where $B$ is the ball having the same center as $Q$ and with

$$
|B|=|E \cap Q| \leqslant 2 C_{1} r^{n^{2} /(n-1)}<\frac{1}{2} r^{n} .
$$

Note that $B$ is strictly contained in $Q$. Since $Q$ is in $A_{\tau}(E)$ and $E$ has no exterior points, we have

$$
0<|E \cap Q| \leqslant 2 C_{1} r^{n^{2} /(n-1)} .
$$

Therefore, by 4.3 ,

$$
\begin{aligned}
C|E \Delta \tilde{E}|^{\beta} & \leqslant C(2|E \cap Q|)^{\beta}=2^{\beta} C|E \cap Q|^{\beta-\alpha}|E \cap Q|^{\alpha} \\
& \leqslant 2^{\beta} C\left(2 C_{1} r^{n^{2} /(n-1)}\right)^{\beta-\alpha}|E \cap Q|^{\alpha}<\tau|E \cap Q|^{\alpha} .
\end{aligned}
$$

Now, $|E|=|\tilde{E}|$ and

$$
\begin{aligned}
\mathbf{T}(\tilde{E}) & =P(\tilde{E}, \Omega)+\mathbf{G}(\tilde{E}) \\
& =P(E, \Omega)-P(E, Q)+\mathcal{H}^{n-1}(E \cap \partial Q)+C_{n}|E \cap Q|^{\alpha}+\mathbf{G}(\tilde{E}) \\
& \leqslant P(E, \Omega)-\tau|E \cap Q|^{\alpha}+\mathbf{G}(\tilde{E}) \quad \text { by } 4.2 \\
& <P(E, \Omega)-C|E \triangle \tilde{E}|^{\beta}+\mathbf{G}(\tilde{E}) \\
& \leqslant P(E, \Omega)+\mathbf{G}(E)=\mathbf{T}(E) .
\end{aligned}
$$

This contradicts the minimality of $E$. Therefore, by Corollary 3.4, only the second case of the corollary can happen here.

Suppose $E$ is a $\mathbf{T}$ minimizer in $\mathcal{F}_{\sigma}$. We may consider another operator

$$
\tilde{\mathbf{T}}(F)=P(F, \Omega)+\tilde{\mathbf{G}}(F)
$$

for any $F \in \mathcal{F}_{1-\sigma}$, where $\tilde{\mathbf{G}}(F)=\mathbf{G}\left(F^{c}\right)$.

Lemma $4.2 E$ is a $\mathbf{T}$ minimizer in $\mathcal{F}_{\sigma}$ if and only if $E^{c}$ is a $\tilde{\mathbf{T}}$ minimizer in $\mathcal{F}_{1-\sigma}$. Moreover, if $\mathbf{G}$ satisfies 4.1 , then so does $\tilde{\mathbf{G}}$ with the same $\beta$.

Proof. This is because

$$
\begin{aligned}
\mathbf{T}(E) \leqslant \mathbf{T}(F) & \Leftrightarrow \mathbf{G}(E)+P(E, \Omega) \leqslant \mathbf{G}(F)+P(F, \Omega) \\
& \Leftrightarrow \tilde{\mathbf{G}}\left(E^{c}\right)+P\left(E^{c}, \Omega\right) \leqslant \tilde{\mathbf{G}}\left(F^{c}\right)+P\left(F^{c}, \Omega\right) \\
& \Leftrightarrow \tilde{\mathbf{T}}\left(E^{c}\right) \leqslant \tilde{\mathbf{T}}\left(F^{c}\right),
\end{aligned}
$$

for any $F \in \mathcal{F}_{\sigma}$. Moreover, if $\mathbf{G}$ satisfies 4.1 , then so does $\tilde{\mathbf{G}}$ with the same $\beta$, because $A \triangle B=$ $A^{c} \triangle B^{c}$. 
From now on, let $E$ be any $\mathbf{T}$ minimizer in $\mathcal{F}_{\sigma}$. To find the regularity of $E$, we adopt the approach given in [2], which corresponds to the case $\mathbf{G} \equiv 0$. A crucial step is to show the existence of both exterior and interior points of the minimizer:

THEOREM 4.3 Let $E$ be any $\mathbf{T}$ minimizer in $\mathcal{F}_{\sigma}$. Then $E$ has both interior points and exterior points in $\Omega$.

Proof. Assume $E$ has no exterior points. Since $0<|E|=\sigma|\Omega|<|\Omega|$, there exists at least one open cube $Q$ in $\Omega$ such that $|E \cap Q|>0$, and also an open ball $B$ in $\Omega$ such that $\left|E^{c} \cap B\right|>0$. We may also require that $Q$ and $B$ are disjoint. Now, by Lemma 4.1. Lemma 4.2 and Remark 3.5. there exist a family $\left\{F_{s}\right\}$ of $n$-dimensional open cubes for $E$ and a family $\left\{K_{s}\right\}$ of $n$-dimensional open balls for $E^{c}$ such that

(1) for each $s>0$, both the edge length of $F_{s}$ and the diameter of $K_{s}$ are equal to $s$;

(2) if $s_{0}$ is the edge length of $Q$ and $t_{0}$ is the diameter of $B$, then $F_{s_{0}}=Q$ and $K_{t_{0}}=B$;

(3) whenever $s<t$, we have $F_{s} \subseteq F_{t}$ and $K_{s} \subseteq K_{t}$;

(4) there exists a decreasing sequence $\left\{s_{i}\right\}_{i=0}^{\infty}$ of positive numbers with limit 0 such that $F_{s_{i}} \in$ $A_{\tau}(E)$ for each $i=1,2, \ldots$

(5) both $\left|F_{s} \cap E\right|$ and $\left|K_{s} \cap E^{c}\right|$ are nondecreasing continuous functions of $s \in(0, \infty)$;

(6) for any $s \leqslant s_{1}$, we have

$$
0<\left|F_{S} \cap E\right| \leqslant C_{E} s^{n^{2} /(n-1)}
$$

for some constant $C_{E}>0$. Also, there exists a positive number $t_{1} \leqslant t_{0}$ such that for any $t \leqslant t_{1}$, we have

$$
0<\left|K_{t} \cap E^{c}\right| \leqslant C_{E^{c}} t^{n^{2} /(n-1)}
$$

for some constant $C_{E^{c}}>0$.

Now, we pick a positive number $\epsilon_{0} \leqslant \min \left\{s_{1}, t_{1}\right\}$ small enough so that

$$
2 n C_{E^{c}}^{1 / n} \epsilon_{0}^{1 /(n-1)} \leqslant \tau
$$

and

$$
\left|F_{\epsilon_{0}} \cap E\right|<\left|B \cap E^{c}\right| .
$$

For any $s \in\left(0, \epsilon_{0}\right)$, since

$$
\left|F_{s} \cap E\right| \leqslant\left|F_{\epsilon_{0}} \cap E\right|<\left|B \cap E^{c}\right|=\left|K_{t_{0}} \cap E^{c}\right|,
$$

by the mean value theorem, there exists at least one $t \leqslant t_{0}$ such that

$$
\left|K_{t} \cap E^{c}\right|=\left|F_{s} \cap E\right| .
$$

Since $E$ has no exterior points, we have $\left|F_{s} \cap E\right|>0$. By the fact that $\lim _{s \rightarrow 0}\left|K_{s} \cap E^{c}\right|=0$, the set of all $t$ satisfying (4.7) must have a minimum in $\left(0, t_{0}\right)$, and we denote this minimum by $g(s)$. Thus, $g(s) \in\left(0, t_{0}\right)$ and

$$
\left|F_{S} \cap E\right|=\left|K_{g(s)} \cap E^{c}\right| .
$$

Note that since $F_{S} \subseteq Q$ and $K_{g(s)} \subseteq K_{t_{0}}=B$, we know that $F_{s}$ and $K_{g(s)}$ are still disjoint. Now, we fix an $s \in\left(0, \epsilon_{0}\right)$ small enough so that $F_{s} \in A_{\tau}(E)$,

$$
\frac{2 n}{\epsilon_{0}} C_{E}^{1 / n} s^{n /(n-1)} \leqslant \tau
$$


and

$$
C\left(C_{E} s^{n^{2} /(n-1)}\right)^{\beta-1+1 / n}<\tau .
$$

For this particular $s$, we consider the set

$$
\tilde{E}=\left(E \backslash F_{s}\right) \cup K_{g(s)} .
$$

Then, by 4.8, we have $|\tilde{E}|=|E|=\sigma|\Omega|$ and

$$
\begin{aligned}
P(\tilde{E}, \Omega)= & P(E, \Omega)-P\left(E, F_{s}\right)+\mathcal{H}^{n-1}\left(E \cap \partial F_{s}\right) \\
& -P\left(E^{c}, K_{g(s)}\right)+\mathcal{H}^{n-1}\left(E^{c} \cap \partial K_{g(s)}\right) .
\end{aligned}
$$

By the isoperimetric inequality [2.1,

$$
P\left(E, F_{S}\right)+\mathcal{H}^{n-1}\left(E \cap \partial F_{S}\right) \geqslant C_{n}\left|E \cap F_{S}\right|^{\alpha} .
$$

Also, by [2.2),

$$
\begin{aligned}
\mathcal{H}^{n-1}\left(E^{c} \cap \partial K_{g(s)}\right)-P\left(E^{c}, K_{g(s)}\right) & \left.\frac{2 n}{g(s)}\left|E^{c} \cap K_{g(s)}\right| \text { by } 4.8\right) \\
& \leqslant \begin{cases}\frac{2 n}{g(s)}\left|E \cap F_{S}\right|^{\alpha} C_{E^{c}}^{1 / n} g(s)^{n /(n-1)} & \text { if } g(s) \leqslant \epsilon_{0}, \text { by } 4.5 \\
\frac{2 n}{g(s)}\left|E \cap F_{S}\right|^{\alpha} C_{E}^{1 / n} s^{n /(n-1)} & \text { if } g(s)>\epsilon_{0}, \text { by } 4.4\end{cases} \\
& \leqslant \begin{cases}\left|E \cap F_{S}\right|^{\alpha} 2 n C_{E^{c}}^{1 / n} \epsilon_{0}^{1 /(n-1)} & \text { if } g(s) \leqslant \epsilon_{0} \\
\left|E \cap F_{S}\right|^{\alpha} \frac{2 n}{\epsilon_{0}} C_{E}^{1 / n} s^{n /(n-1)} & \text { if } g(s)>\epsilon_{0}\end{cases} \\
& \leqslant \tau\left|E \cap F_{S}\right|^{\alpha} \quad \text { by (4.9) and (4.6). }
\end{aligned}
$$

Therefore, by (4.11), the fact that $F_{s} \in A_{\tau}(E)$, and 3.4 , we have

$$
\begin{aligned}
P(\tilde{E}, \Omega) & \leqslant P(E, \Omega)-C_{n}\left|E \cap F_{s}\right|^{\alpha}+2 \mathcal{H}^{n-1}\left(E \cap \partial F_{s}\right)+\tau\left|E \cap F_{s}\right|^{\alpha} \\
& \leqslant P(E, \Omega)-C_{n}\left|E \cap F_{s}\right|^{\alpha}+3 \tau\left|E \cap F_{S}\right|^{\alpha} \leqslant P(E, \Omega)-\tau\left|E \cap F_{S}\right|^{\alpha},
\end{aligned}
$$

due to 3.5 . Hence,

$$
\begin{aligned}
\mathbf{T}(\tilde{E}) & =P(\tilde{E}, \Omega)+\mathbf{G}(\tilde{E}) \\
& \leqslant P(E, \Omega)-\tau\left|E \cap F_{s}\right|^{\alpha}+\mathbf{G}(E)+C\left|E \cap F_{S}\right|^{\beta} \\
& =\mathbf{T}(E)+\left|E \cap F_{s}\right|^{\alpha}\left(C\left|E \cap F_{s}\right|^{\beta-1+1 / n}-\tau\right)<\mathbf{T}(E) .
\end{aligned}
$$

The last inequality follows from 4.4 and 4.10 . This contradicts the $\mathbf{T}$ minimality of $E$. Therefore, $E$ must have exterior points. Since, by Lemma $4.2, E^{c}$ is a $\tilde{\mathbf{T}}$ minimizer, we see that $E^{c}$ also has some exterior points. Therefore, $E$ has both interior points and exterior points. 


\subsection{Regularity results}

Now, we may discuss the regularity of the $\mathbf{T}$ minimizer $E$. By Theorem $4.3, E$ has both interior points and exterior points. Therefore, there exists a number $R>0$ and two open balls $B_{1}, B_{2}$ in $\Omega$ of the same radius $2 R$ such that

$$
\left|E \cap B_{1}\right|=0 \text { and }\left|E^{c} \cap B_{2}\right|=0 .
$$

Our main theorem is stated as follows.

Theorem 4.4 Suppose $E$ is a minimizer of the quasi perimeter $\mathbf{T}$ in $\mathcal{F}_{\sigma}$. Then $E$ is a quasi minimizer of perimeter (without the volume constraint) in the sense that

$$
P(E, \Omega) \leqslant P(F, \Omega)+c|E \triangle F|^{\min (1, \beta)}
$$

for all subsets $F$ of $\Omega$ with $E \triangle F$ contained in any open ball $B_{\rho}$ with radius $\rho<R$, and for some constant $c>0$.

Proof. By Theorem 4.3 there exist two open balls $B_{1}$ and $B_{2}$ in $\Omega$ of the same radius $2 R$ such that

$$
\left|E \cap B_{1}\right|=0 \text { and }\left|E^{c} \cap B_{2}\right|=0 .
$$

Let $\rho \in(0, R)$ be any fixed number and $F$ be any set of finite perimeter with $E \triangle F$ contained in some open ball $B_{\rho}$ of radius $\rho$.

Suppose that $\left|E \cap B_{\rho}\right| \geqslant\left|F \cap B_{\rho}\right|$. We can move a ball $B$ in $B_{1} \backslash B_{\rho}$ of radius $R$, while keeping it strictly in $\Omega \backslash \bar{B}(\rho)$, until it reaches a new position $B_{3}$ such that

$$
\left|B_{3} \cap E^{c}\right|=\left|E \cap B_{\rho}\right|-\left|F \cap B_{\rho}\right| .
$$

Let $F_{\rho}=F \cup B_{3}$. Then $\left|F_{\rho}\right|=|E|$. Since $E$ is a $\mathbf{T}$ minimizer, we get

$$
\mathbf{T}(E) \leqslant \mathbf{T}\left(F_{\rho}\right) .
$$

That is,

$$
\mathbf{G}(E)+P(E, \Omega) \leqslant \mathbf{G}\left(F_{\rho}\right)+P\left(F_{\rho}, \Omega\right) .
$$

Therefore, by 2.2,

$$
\begin{aligned}
P(E, \Omega) & \leqslant \mathbf{G}\left(F_{\rho}\right)-\mathbf{G}(E)+P\left(F_{\rho}, \Omega\right) \\
& \leqslant C\left|E \triangle F_{\rho}\right|^{\beta}+P(F, \Omega)-P\left(B_{3} \cap E^{c}, B_{3}\right)+\mathcal{H}^{n-1}\left(\partial B_{3} \cap E^{c}\right) \\
& \leqslant P(F, \Omega)+C\left|E \triangle F_{\rho}\right|^{\beta}+\frac{n}{R}\left|B_{3} \cap E^{c}\right| \\
& \leqslant P(F, \Omega)+c|E \triangle F|^{\min (1, \beta)},
\end{aligned}
$$

where $c=C+n / R$.

We arrive at the same relation

$$
P(E, \Omega) \leqslant P(F, \Omega)+c|E \Delta F|^{\min (1, \beta)}
$$

if we suppose $\left|E \cap B_{\rho}\right| \leqslant\left|F \cap B_{\rho}\right|$. 
By the theorem and a well known result about quasi minimizers of perimeter satisfying (4.12) (see for instance [4, Theorem 1]), we get the desired classical regularity result for the boundary of $E$ as follows:

THEOREM 4.5 Suppose $E$ is a minimizer of the quasi perimeter $\mathbf{T}$ in $\mathcal{F}_{\sigma}$. Then the reduced boundary $\partial^{*} E$ is an $(n-1)$-dimensional $C^{1, \min (1 / 2, \beta / 2)}$ hypersurface in $\Omega$, and moreover we have $\operatorname{dim}\left(\left(\partial E \backslash \partial^{*} E\right) \cap \Omega\right) \leqslant n-8$.

\section{REFERENCES}

1. Evans, L. C. \& GARIEPY, R. Measure Theory and Fine Properties of Functions. Stud. Adv. Math., CRC Press (1992). Zbl 0804.28001 MR 1158660

2. Gonzalez, E., Massari, U., \& TAmanini, I. On the regularity of boundaries of sets minimizing perimeter with a volume constraint. Indiana Univ. Math. J. 32 (1983), 25-37. Zbl 0486.49024 MR 0684753

3. MASSARI, U. Frontiere orientate di curvatura media assegnata in $L^{p}$. Rend. Sem. Mat. Univ. Padova 53 (1975), 37-52. Zbl 0358.49019 MR 0417905

4. Tamanini, I. Boundaries of Caccioppoli sets with Hölder-continuous normal vector. J. Reine Angew. Math. 334 (1982), 27-39. Zbl 0479.49028 MR 0667448

5. Villani, C. Topics in Optimal Transportation. Grad. Stud. Math. 58, Amer. Math. Soc. (2003). Zbl pre01909499 MR 1964483 